\title{
Publisher Correction: Costs and consequences of wind turbine wake effects arising from uncoordinated wind energy development
}

J. K. Lundquist (iD, K. K. DuVivier, D. Kaffine and J. M. Tomaszewski

Correction to: Nature Energy https://doi.org/10.1038/s41560-018-0281-2, published online 26 November 2018.

In the version of this Article originally published, the labels for the colour scales in Fig. 4 were mistakenly swapped. The label in panel a should have read 'Power deficit (MW)' and the label in panel b should have read 'Wind speed deficit ( $\left.\mathrm{m} \mathrm{s}{ }^{-1}\right)$ '. This has now been corrected.

Published online: 5 March 2019

https://doi.org/10.1038/s41560-019-0363-9 